\title{
Determination of viscosity and pressure in an elastohydrodynamic rolling contact by using optical interferometry: a theoretical study
}

J O Östensen, PhD, R Larsson, PhD

Division of Machine Elements, Luleå University of Technology, Luleå, Sweden

C H Venner, PhD

Faculty of Mechanical Engineering, University of Twente, Enschede, The Netherlands

Optical interferometry has been used during the last 30 years for film thickness measurements in elastohydrodynamic (EHD) contacts. In the work presented in this paper the possibility of using optical interferometry for determining pressure and apparent viscosity in a fully flooded EHD point contact has been theoretically investigated. The pressure in the contact is computed from elastic deformation theory and force balance. The apparent viscosity can be obtained from the Reynolds equation when the film thickness and the pressure are known. To theoretically examine the sensitivity to measurement errors, different errors have been implemented in a set of 'exact' numerical data.

From the theoretical study it can be seen that a film thickness map obtained from optical interferometry can be used for determination of both pressure and apparent viscosity measurement in an EHD point contact. The simulated errors that have been used do not influence the pressure within the Herzian region by more than a few per cent. However, to obtain reliable results from the apparent viscosity calculations, the measurements of film thickness, load and elastic modulus must be very accurate. It is seen, for example, that the resolution of the film thickness measurement has to be less than $1 \mathrm{~nm}$ to achieve high accuracy.

Key words: film thickness, EHD, point contact, pressure distribution, optical interferometry, apparent viscosity

\section{NOTATION}

$b_{\mathrm{H}} \quad$ Hertzian radius $b_{\mathrm{H}}^{3}=3 w R /\left(2 E^{\prime}\right)(\mathrm{m})$

$E^{\prime} \quad$ effective elastic modulus $=2\left[\left(1-v_{1}^{2}\right) / E_{1}\right.$ $\left.+\left(1-v_{2}^{2}\right) / E_{2}\right]^{-1}(\mathrm{~Pa})$

$G \quad$ dimensionless material parameter $=\alpha E^{\prime}$

$h \quad$ film thickness (m)

$h_{\mathrm{c}} \quad$ central film thickness (m)

$h_{0}$ rigid separation constant in the film thickness equation (m)

$\Delta h \quad$ resolution of film thickness in interferometry measurements (m)

$p$

$p_{\mathbf{H}}$

$p_{0}$

$R$

$\eta_{0} \quad$ Viscosity at ambient pressure $(\mathrm{Pa} \mathrm{s})$

$\bar{\eta} \quad$ dimensionless apparent viscosity $=\eta / \eta_{0}$

$\lambda \quad$ wavelength (m)

$v \quad$ Poisson's ratio

The MS was received on 2 May 1995 and was accepted for publication on 5 March 1996

$J 01795$ C IMechE 1996 $\rho \quad$ density $\left(\mathrm{kg} / \mathrm{m}^{3}\right)$

$\rho_{0} \quad$ density at ambient pressure $\left(\mathrm{kg} / \mathrm{m}^{3}\right)$

$\Delta \phi \quad$ difference in phase change (rad)

\section{INTRODUCTION}

Optical interferometry methods have been used during the last 30 years for film thickness measurements in elastohydrodynamic (EHD) contacts. The first significant publication on this topic was Cameron and Gohar (1) in 1966. In 1968 Foord et al. (2) improved the procedure in many ways; for example they used a coated layer on the glass disc allowing a better interference pattern. They also computed the Barus pressureviscosity coefficient for different lubricating oils by using their empirical film thickness equation.

Paul and Cameron (3) in 1972 used an impact microviscosimeter to evaluate both the pressure distribution and the apparent viscosity of a lubricant in an EHD point contact at pure squeeze. They used optical interferometry to obtain a film thickness map of the contact. When the deformation of the surfaces was known, the pressure in the whole contact could be derived from the inverse elasticity theory. The Reynolds equation was used to derive the apparent viscosity of the tested lubricant. Wong et al. (4-6) have continued Paul and Cameron's work. They have further developed the impact microviscosimeter technique to give true shear stress and true shear rate. They also used image processing to obtain the film thickness profile.

In 1994 Gustafsson et al. (7) developed an image processing method for the analysis of the film thickness in an EHD point contact. This image processing method makes it possible to extract a $2048 \times 3072$ pixel film thickness map and was later used by Åström and 
Venner (8). They used the elastic deformation theory and the force balance to compute the pressure distribution in a grease-lubricated point contact at pure rolling.

A film thickness map obtained from optical interferometry measurements of a fully flooded EHD point contact can be used to compute the pressure distribution and the apparent viscosity. In the work presented in this paper a theoretical investigation has been made to find out how sensitive the computed pressure distribution and the apparent viscosity are to different experimental measurement errors by implementing simulated measurement errors in a set of 'exact' data, for example to determine the effect of an error in the refractive index. The 'exact' pressure distribution and film thickness are results from a numerical solution of the EHD point contact problem. The results from this investigation are thought to provide a guideline for future experimental studies.

\section{THEORY}

\subsection{Pressure calculation}

A ball and disc apparatus is often used to simulate the elastohydrodynamic contact between two nonconformal surfaces such as a ball and an inner ring of a ball-bearing. Optical interferometry is used to measure the lubricant film thickness between the ball and the flat glass disc. From the film thickness map of this circular contact it is possible to find out the sum of surface deformations of the ball and the disc. The deformation is the difference between the measured film thickness and the geometrical separation which is given by the shape of the ball.

If the deformation is known, it is also possible to find out the magnitude and the distribution of the pressure that causes this deformation. The film thickness equation can be written as

$$
h(x, y)=h_{0}+g(x, y)+d(x, y)
$$

where $h_{0}$ is a constant, $g$ is the undeformed geometry of the ball and $d$ is the total elastic deformation of the two surfaces. If the spherical shape of the ball is approximated by paraboloids and the surfaces are assumed to be perfectly smooth, the separation can be obtained as

$$
g(x, y)=\frac{x^{2}+y^{2}}{2 R}
$$

The expression for the deformation can be found if both bodies are approximated by elastic half-spaces as

$$
d(x, y)=\frac{2}{\pi E^{\prime}} \int_{-\infty}^{\infty} \int_{-\infty}^{\infty} \frac{p\left(x^{\prime}, y^{\prime}\right) \mathrm{d} x^{\prime} \mathrm{d} y^{\prime}}{\sqrt{\left[\left(x-x^{\prime}\right)^{2}+\left(y-y^{\prime}\right)^{2}\right]}}
$$

The influences of ball curvature and friction forces in the contact are very small and are thus neglected in equation (3). The constant $h_{0}$ is determined by solving the force balance equation:

$$
w=\int_{-\infty}^{\infty} \int_{-\infty}^{\infty} p(x, y) d x d y
$$

The pressure along the boundaries is set to zero and the cavitation condition $p \geqslant 0$ is applied in the cavitated region.

Part J: Journal of Engineering Tribology

\subsection{Apparent viscosity}

When both film thickness and pressure are known it is possible to calculate the lubricant viscosity variation in the contact region. An apparent viscosity can be obtained from the Reynolds equation:

$$
\frac{\partial}{\partial x}\left(\frac{\rho h^{3}}{12 \eta} \frac{\partial p}{\partial x}\right)+\frac{\partial}{\partial y}\left(\frac{\rho h^{3}}{12 \eta} \frac{\partial p}{\partial y}\right)=\frac{u}{2} \frac{\partial}{\partial x}(\rho h)
$$

where the unknowns are viscosity and density. Density can be measured or calculated from some appropriate expression such as the Dowson-Higginson pressuredensity relationship (9):

$$
\rho=\rho_{0}\left(\frac{0.59 \times 10^{9}+1.35 p}{0.59 \times 10^{9}+p}\right)
$$

The thermal effect on the apparent viscosity is minimized since pure rolling is assumed. The different experimental parameters such as load, rolling velocity and ball radius can be chosen to minimize the inlet shear heating.

\section{SOLUTION TECHNIQUE}

A theoretical analysis of the sensitivity to different sources of error has been carried out to check the possibility of computing the contact pressure from equations (1) and (4) and the apparent viscosity from equation (5). A numerical solution of equations (1), (4) and (5) has been used to obtain a reference film thickness map. The film thickness map, referred to as the 'exact' film thickness, is solved on a $513 \times 513$ grid by using multi-level techniques $(10,11)$. The 'exact' pressure distribution is also received from the numerical solution. The assumptions made to compute the exact film thickness map were isothermal conditions, Newtonian lubricant, density according to equation (6) and a pressureviscosity relationship according to Roelands (12):

$$
\eta(p)=\eta_{0} \exp \left\{\left(9.67+\ln \eta_{0}\right)\left[-1+\left(1+\frac{p}{p_{0}}\right)^{2}\right]\right\}
$$

\subsection{Solution of pressure and apparent viscosity}

The pressure distributions for the studied cases are solved from equations (1) and (4) in the same way as described by Aström and Venner (8). The multi-grid technique is used to increase the convergence rate of the relaxation of the non-linear equations.

By the use of the chain rule, the first term of equation (5) can be written as

$$
\begin{aligned}
\frac{\partial}{\partial x}\left(\frac{\rho h^{3}}{12 \eta} \frac{\partial p}{\partial x}\right)= & \frac{\partial}{\partial x}\left(\frac{\varepsilon_{0}}{\bar{\eta}} \frac{\partial p}{\partial x}\right) \\
= & -\frac{1}{\bar{\eta}^{2}} \frac{\partial \bar{\eta}}{\partial p} \varepsilon_{0}\left(\frac{\partial p}{\partial x}\right)^{2} \\
& +\frac{1}{\bar{\eta}}\left(\varepsilon_{0} \frac{\partial^{2} p}{\partial x^{2}}+\frac{\partial \varepsilon_{0}}{\partial x} \frac{\partial p}{\partial x}\right)
\end{aligned}
$$

where

$$
\varepsilon_{0}=\frac{\rho h^{3}}{12 \eta_{0}}, \quad \bar{\eta}=\frac{\eta}{\eta_{0}}
$$


If the second term in equation (5) is treated in a similar way, the Reynolds equation becomes

$$
\begin{aligned}
& -\frac{1}{\bar{\eta}^{2}} \frac{\partial \bar{\eta}}{\partial \bar{p}} \varepsilon_{0}\left[\left(\frac{\partial p}{\partial x}\right)^{2}+\left(\frac{\partial p}{\partial y}\right)^{2}\right] \\
& +\frac{1}{\bar{\eta}}\left[\varepsilon_{0}\left(\frac{\partial^{2} p}{\partial x^{2}}+\frac{\partial^{2} p}{\partial y^{2}}\right)+\frac{\partial \varepsilon_{0}}{\partial x} \frac{\partial p}{\partial x}+\frac{\partial \varepsilon_{0}}{\partial y} \frac{\partial p}{\partial y}\right]=\frac{u}{2} \frac{\partial}{\partial x}(\rho h)
\end{aligned}
$$

which can be rewritten as

$$
\frac{\partial \bar{\eta}}{\partial p}=c_{1} \bar{\eta}-c_{2} \bar{\eta}^{2}
$$

where

$$
\begin{aligned}
& c_{1}(x, y)=\frac{\varepsilon_{0}\left(\frac{\partial^{2} p}{\partial x^{2}}+\frac{\partial^{2} p}{\partial y^{2}}\right)+\frac{\partial \varepsilon_{0}}{\partial x} \frac{\partial p}{\partial x}+\frac{\partial \varepsilon_{0}}{\partial y} \frac{\partial p}{\partial y}}{\varepsilon_{0}\left[\left(\frac{\partial p}{\partial x}\right)^{2}+\left(\frac{\partial p}{\partial y}\right)^{2}\right]} \\
& c_{2}(x, y)=\frac{\frac{u}{2} \frac{\partial}{\partial x}(\rho h)}{\varepsilon_{0}\left[\left(\frac{\partial p}{\partial x}\right)^{2}+\left(\frac{\partial p}{\partial y}\right)^{2}\right]}
\end{aligned}
$$

The functions $c_{1}$ and $c_{2}$ can be evaluated at each point $(x, y)$ if the pressure, film thickness, viscosity at ambient pressure, density and velocity sum are known. This means that the dimensionless apparent viscosity in one single point can be found from equation (9) without solving a complete set of finite difference equations. All derivatives in $c_{1}$ and $c_{2}$ are computed by using central differences.

It is reasonable to use the exponential expression of the Roelands pressure-viscosity relationship (7) as a solution, $\bar{\eta}(p)$, to equation (9). Inserted into equation (9) it becomes

$$
\begin{aligned}
& \frac{\left(9.67+\ln \eta_{0}\right) z}{p_{0}}\left(1+\frac{p}{p_{0}}\right)^{z-1}-c_{1}+c_{2} \\
& \times \exp \left\{\left(9.67+\ln \eta_{0}\right)\left[-1+\left(1+\frac{p}{p_{0}}\right)^{z}\right]\right\}=0
\end{aligned}
$$

The pressure-viscosity index, $z$, can be obtained from equation (10) at each grid point. If the Roelands pressure-viscosity relationship is valid this pressureviscosity index should be the same at each grid point. However, if it is not or if any errors in the measurements exists, it will vary from point to point. In the more general case it is, of course, more realistic to assume some general expression for $\bar{\eta}(p)$. This general expression may include more than one constant, which have to be determined at each grid point. In that case the optimum values of the constants can be calculated by means of a least-squares fit of $\bar{\eta}(p)$ to equation (9) at several grid points at the same time.

\subsection{Sources of error}

Several possible sources of error are present when the film thickness is measured by using optical interferometry. To transform optical path length to geometrical path length, that is film thickness, it is essential to know the refractive index, $n$, of the lubricant and the differ- ence in phase change, $\Delta \phi$, on reflection in the chromium layer and in the steel ball surface. If the wrong value of the refractive index is used the film thickness map includes an error which is proportional to the error in the refractive index. If a wrong value of the difference in phase change on reflection is used, a constant error term is added or subtracted to the film thickness, as shown by the following equation:

$$
h=\frac{\lambda}{2 n}\left(q+\frac{\Delta \phi}{2 \pi}\right)
$$

When the pressure is solved from equations (1) and (4) additional errors may occur. The effective elastic modulus and the load have to be known. It is also important to find the curvature centre of the ball, that is $x=0$ and $y=0$, in the film thickness map.

The resolution in film thickness in the interferometry measurements may also influence pressure and viscosity calculations. The resolution, $\Delta h$, is the smallest difference in film thickness that is possible to detect and originates from the resolution in the hue measurements (7). The resolution causes a truncation of the true film thickness which may give rise to errors in both the solution of the pressure distribution [equations (1) and (4)] and in the solution of apparent viscosity [equation (5)].

Errors in the viscosity at ambient pressure, $\eta_{0}$, and in the velocity sum, $u$, do not give rise to errors in film thickness or pressure, but they influence the solution for apparent viscosity.

To simulate the effect of the sources of error described above, these errors are introduced in the exact film thickness map and in the effective elastic modulus, in the load, in the viscosity at ambient pressure and in the velocity sum. Then the pressure viscosity index, $z$, is obtained from equation (10).

\section{RESULTS AND DISCUSSION}

\subsection{Exact solution}

The 'exact' solution is computed by using material properties, geometrical properties and running properties according to Table 1 . This corresponds to a normal test in a ball and disc apparatus with an ordinary oil as lubricant and a disc made of glass.

The pressure-viscosity index, $z$, in Table 1 was calculated as $\alpha p_{0} /\left(\ln \eta_{0}+9.67\right)$. The dimensionless speed parameter is $U=1.3 \times 10^{-11}$, the material parameter is $G=2420$ and the load parameter is $W=4.95 \times 10^{-6}$. These data have been used to numerically solve the film thickness and the pressure distribution on a $513 \times 513$ grid from equations (1), (4)

Table 1 Data for the exact solution

\begin{tabular}{ccl}
\hline Parameter & \multicolumn{1}{c}{ Value } & Dimension \\
\hline$E^{\prime}$ & $1.1 \times 10^{11}$ & $\mathrm{~Pa}$ \\
$\alpha$ & $2.2 \times 10^{-8}$ & $\mathrm{~Pa}^{-1}$ \\
$\eta_{\mathrm{o}}$ & $59.5 \times 10^{-3}$ & $\mathrm{~Pa} \mathrm{~s}$ \\
$\rho_{\mathrm{O}}$ & 913 & $\mathrm{~kg} / \mathrm{m}^{3}$ \\
$w$ & 340 & $\mathrm{~N}$ \\
$u$ & 1.2 & $\mathrm{~m} / \mathrm{s}$ \\
$R$ & 0.025 & $\mathrm{~m}$ \\
$b_{\mathrm{H}}$ & $488 \times 10^{-6}$ & $\mathrm{~m}$ \\
$p_{\mathrm{H}}$ & $683 \times 10^{6}$ & $\mathrm{~Pa}$ \\
$z$ & 0.636 & - \\
\hline
\end{tabular}

Proc Instn Mech Engrs Vol 210 
and (5). The maximum residual in the solution of the Reynolds equation is $O\left(10^{-6}\right)$ and the maximum residual of the force balance is $O\left(10^{-10}\right)$. The minimum film thickness and the central film thickness are in this case $h_{\min }=0.13 \mu \mathrm{m}$ and $h_{\mathrm{c}}=0.33 \mu \mathrm{m}$.

\subsection{Effect of mesh}

The mesh size is varied to study the influence on the solution for apparent viscosity in the case of no implemented errors. Equation (10) is solved in a narrow region along the $x$ axis $(-0.55<x<0.29 \mathrm{~mm}$ ) (see Fig. 1), where the exact film thickness and the exact pressure distribution are used. This narrow region is chosen to give a variety of pressures ranging from almost zero to maximum pressure. To extract the coarser grid film thickness and pressure distribution, the injection method is used, that is coarse grid data are the same as in the coinciding fine grid points.

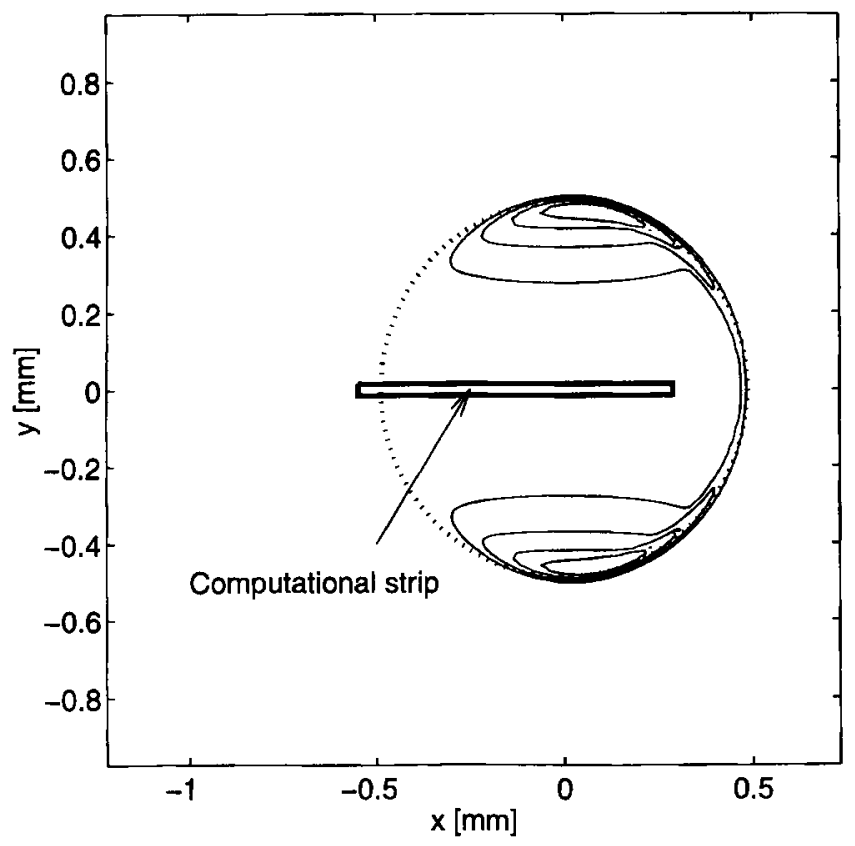

Fig. 1 Film thickness map and area of computation
It is seen from Fig. 2 that even with a $513 \times 513$ grid it is not possible to achieve the correct viscosity at high pressures. The reason for this is that the terms $c_{1}$ and $c_{2}$ in equation (9) approach infinity when the pressure derivatives in both $x$ and $y$ directions approach zero, as they do in the centre of the contact. The sensitivity to numerical errors is therefore very high at the contact centre. In the contact centre, that is at high pressures, it is sometimes impossible to find any solution to equation (10). This problem is worst for the coarse grid cases. For finer grids it is possible to find solutions to equation (10), but these may be quite inaccurate. That is the reason why the accuracy seems to be worse for finer grids.

From the results in Fig. 2 it is possible to conclude that a grid size of $129 \times 129$ will give a reasonably good result for the computation of apparent viscosity. This grid size also gives a more reasonable computing time when computing the deformation in the contact.

\subsection{Errors introduced into the solution}

To simulate an error in load and effective elastic modulus, they are varied within a few per cent of their correct values. An error in the phase change difference is the same as a few per cent error in the central film thickness, which is added to or subtracted from the correct film thickness. To simulate an error in the refractive index, the complete film thickness map is multiplied by a constant. The resolution, $\Delta h$, of the film thickness is chosen to be 1 or $10 \mathrm{~nm}$ and the centring error of the contact is some percentage of $b_{\mathrm{H}}$ in the positive $x$ and $y$ directions. The magnitude of the errors can be seen in Table 2 .

Table 2 Introduced errors

\begin{tabular}{ll}
\hline Parameters & \multicolumn{1}{c}{ Error } \\
\hline$w$ and $E^{\prime}$ & 1 and $5 \%$ \\
$n$ & 1 and $5 \%$ of $h(x, y)$ \\
$\Delta \phi$ & 1 and $5 \%$ of $h_{\mathrm{c}}$ \\
Centring & 1 and $5 \%$ of $b_{\mathrm{H}}$ in both $x$ and $y$ directions \\
Resolution & 1 and $10 \mathrm{~nm}$ \\
$\eta_{0}$ and $u$ & 20 and $50 \%$ \\
\hline
\end{tabular}
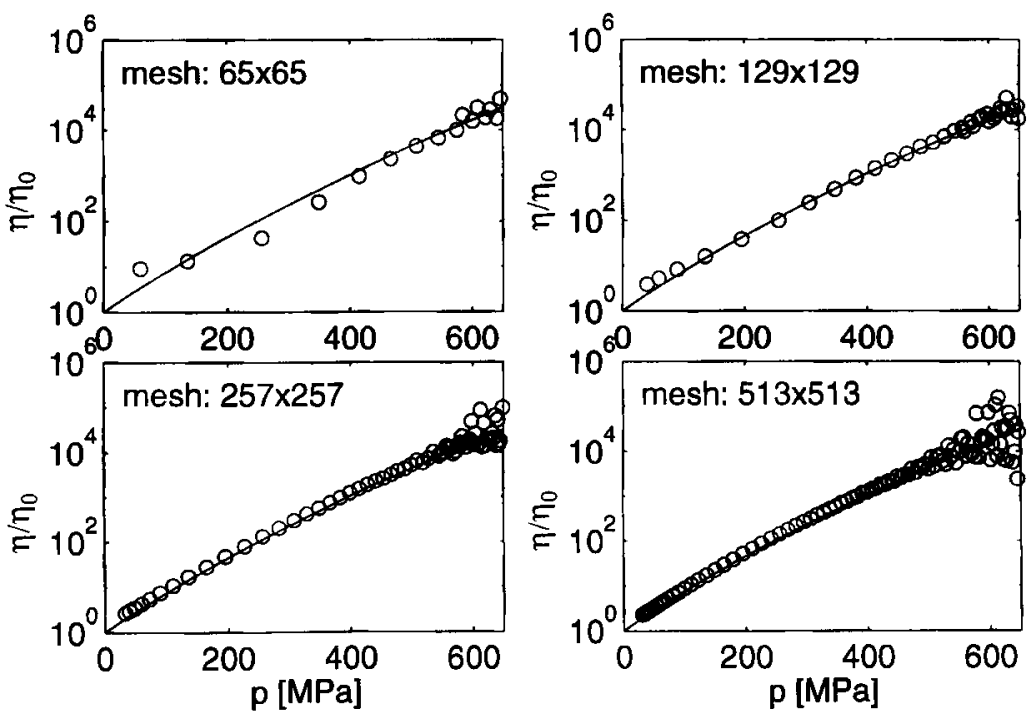

Fig. 2 Effect of mesh size on the dimensionless apparent viscosity 


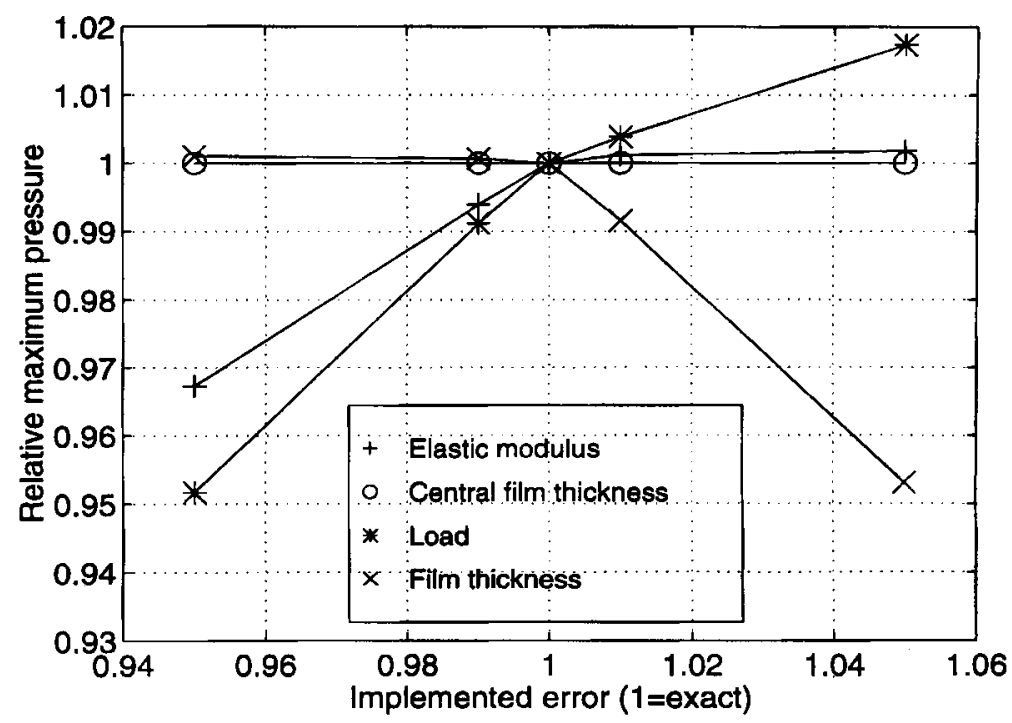

Fig. 3 Relative maximum pressure with implemented errors

\subsection{Effect on pressure}

The solution of the pressure distribution from equations (1) and (4) will be affected by the implemented errors. Figure 3 shows how the maximum pressure is influenced by the different artificial defects. It is seen that the effective elastic modulus influences maximum pressure very little as long as it is larger than the true value. The expression for the maximum Hertzian pressure in the non-lubricated case is

$$
p_{\mathrm{H}}=\frac{1}{\pi}\left(\frac{3 w E^{\prime 2}}{2 R^{2}}\right)^{1 / 3}
$$

The reason why the maximum pressure does not increase as much as stated in equation (12) is that a large erroneous pressure occurs along the boundaries of the computational domain when $E^{\prime}$ is too large (see Fig. 4). This erroneous pressure occurs since the film thickness map does not match the deformation which corresponds to the erroneous effective elastic modulus. To fulfil the force balance equation (4), a pressure has to develop along the boundaries. The maximum pressure follows equation (12) well when the effective elastic modulus is too small. Figure 5a shows how the pressure varies along the $x$ axis.

A similar effect is seen when an error is implemented in the load (see Figs 3 and $5 b$ ). If the load is too small, an erroneous pressure occurs along the boundaries of the computational domain, and thus the maximum pressure will be lower than the corresponding Hertzian pressure. When the load is too large it follows equation (12) well.

From Figs 3 and $5 \mathrm{c}$ it is seen that an overall error of -5 per cent in film thickness has very little influence on the pressure. The effect on the pressure, is, however, larger if the film thickness is greater than its true values. The reason for this is the computation domain boundary pressure which occurs to fulfil the force balance equation when the gap is too large.

Errors in the central film thickness do not influence the pressure at all. This is an effect of the choice of elastic half-spaces. It is only the shape of the deformed surfaces that contributes to the pressure distribution and not the absolute value of the gap.

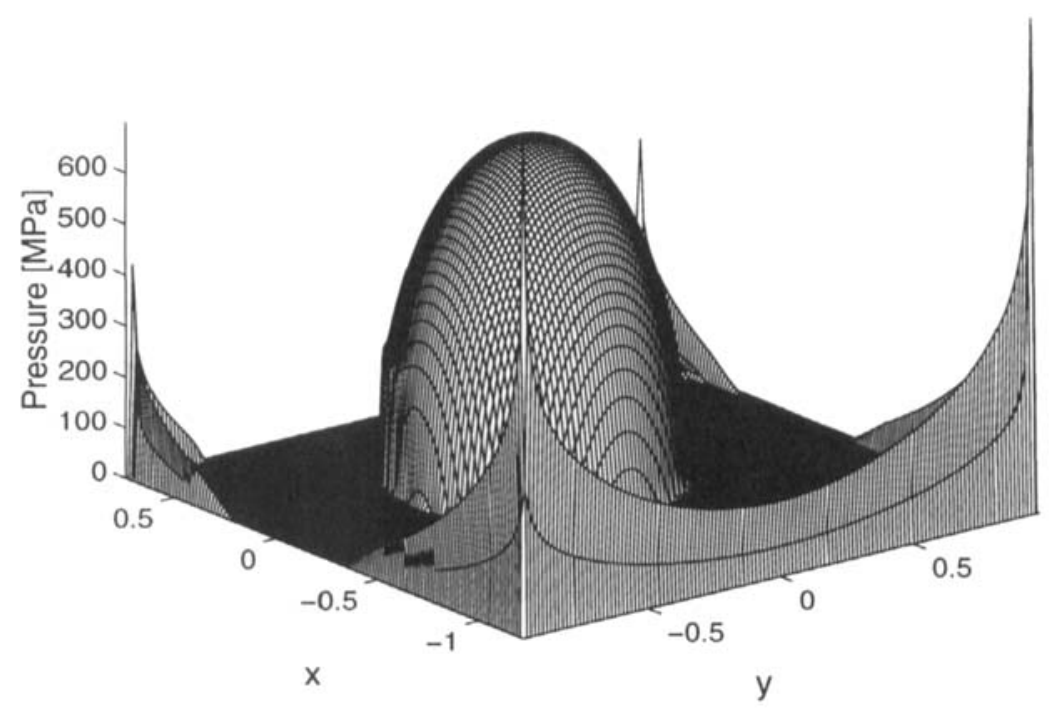

Fig. 4 Pressure distribution for the case of too large an effective elastic modulus 

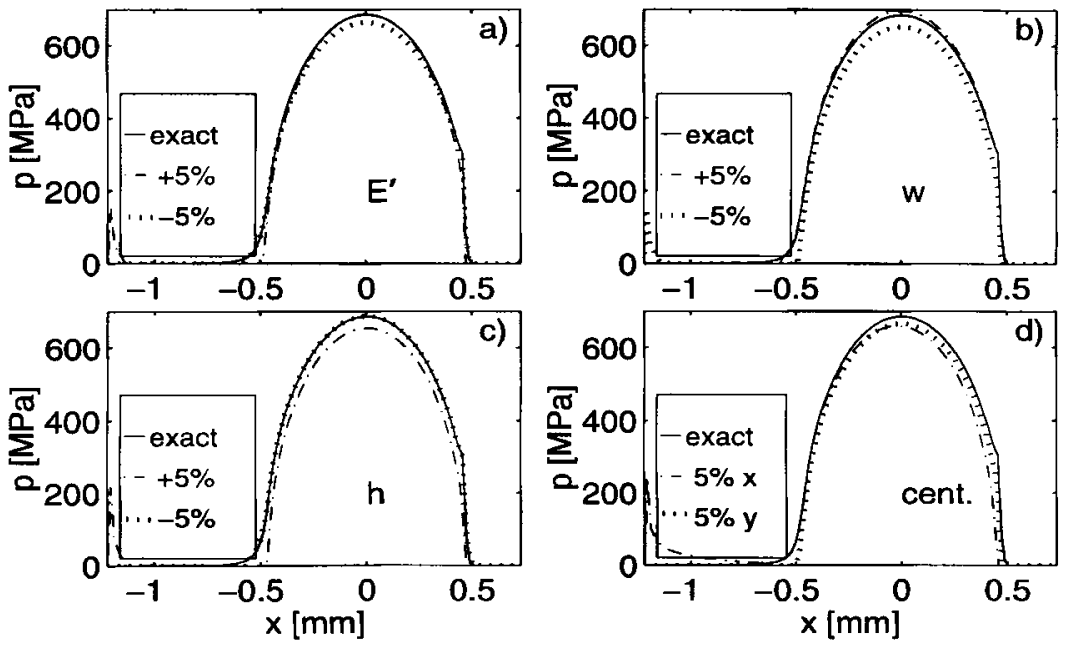

Fig. 5 Pressure distributions with implemented errors: (a) error in effective elastic modulus, (b) error in load, (c) error in film thickness, (d) error in centring

Table 3 Effect of errors in the centre position on the maximum pressure

\begin{tabular}{lc}
\hline Error & Relative maximum pressure \\
\hline $1 \%$ of $b_{\mathbf{H}}$ in the $x$ direction & 0.995 \\
$5 \%$ of $b_{\mathbf{H}}$ in the $x$ direction & 0.962 \\
$1 \%$ of $b_{\mathbf{H}}$ in the $y$ direction & 0.995 \\
$5 \%$ of $b_{\mathbf{H}}$ in the $y$ direction & 0.970 \\
\hline
\end{tabular}

Table 3 shows the effects of bad centring of the ball's curvature centre on the maximum pressure. The maximum pressure will be too low in all these cases since an erroneous pressure occurs along one of the boundaries of the computational domain. Figure 5d shows the pressure along the $x$ axis. The truncation of the film thickness due to a limited resolution of 1 or 10 $\mathrm{nm}$ has a very small effect on the pressure distribution.

\subsection{Effect on dimensionless apparent viscosity}

The next step is to investigate how the different errors influence the apparent viscosity. The pressure viscosity index, $z$, is obtained from equation (10) in the domain shown in Fig. 1. Figures 6 to 11 show how the dimensionless apparent viscosity, $\bar{\eta}=\eta / \eta_{0}$, is affected by the implemented errors. It is seen that numerical problems occur at high pressure in all the different cases. This is because of the singularity in the terms $c_{1}$ and $c_{2}$ at the contact centre, that is where the pressure reaches its maximum. The Couette term $\partial(\rho h) / \partial x$ needs to be determined very accurately to get a good estimation of $c_{2}$, but this is very difficult in the contact centre where it is close to zero.

It is also seen from all cases that the dimensionless apparent viscosity at low pressure, that is just outside the Hertzian region, does not follow the Roelands relationship very well. This is caused by the injection from the fine $513 \times 513$ grid to the coarser $129 \times 129$ grid. The rapid change of the Couette term of equation (5) in this region is not fully resolved on the coarser grid. The results are thus only reliable for a pressure between approximately $100-500 \mathrm{MPa}$ in this case. Reliable results could probably be obtained for higher pressure in a higher load case.

Figure 6 shows the dimensionless apparent viscosity for different erroneous values of the effective elastic

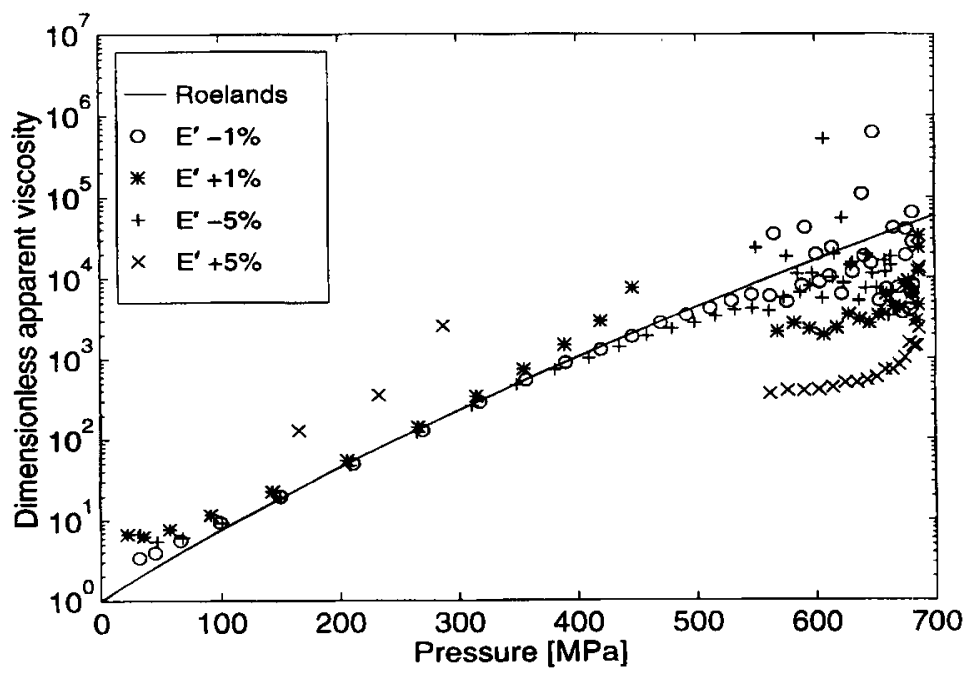

Fig. 6 Effect of error in effective elastic modulus, $E^{\prime}$, on the dimensionless apparent viscosity 
modulus, $E^{\prime}$. Small effects on the dimensionless apparent viscosity can be seen if $E^{\prime}$ is less than its true value. The pressure distribution with a 5 per cent reduction in $E^{\prime}$ follows the exact pressure distribution quite well up to $400-500 \mathrm{MPa}$. If the effective elastic modulus is greater than the true value, the pressure will differ more from the exact pressure, giving large effects on the dimensionless apparent viscosity (Fig. 5a).

Similar effects are seen if a load error is introduced (see Fig. 7). If the load is too small the solution of the pressure-viscosity index will contain large errors.

Figures 8 and 9 show the effect of errors in the phase

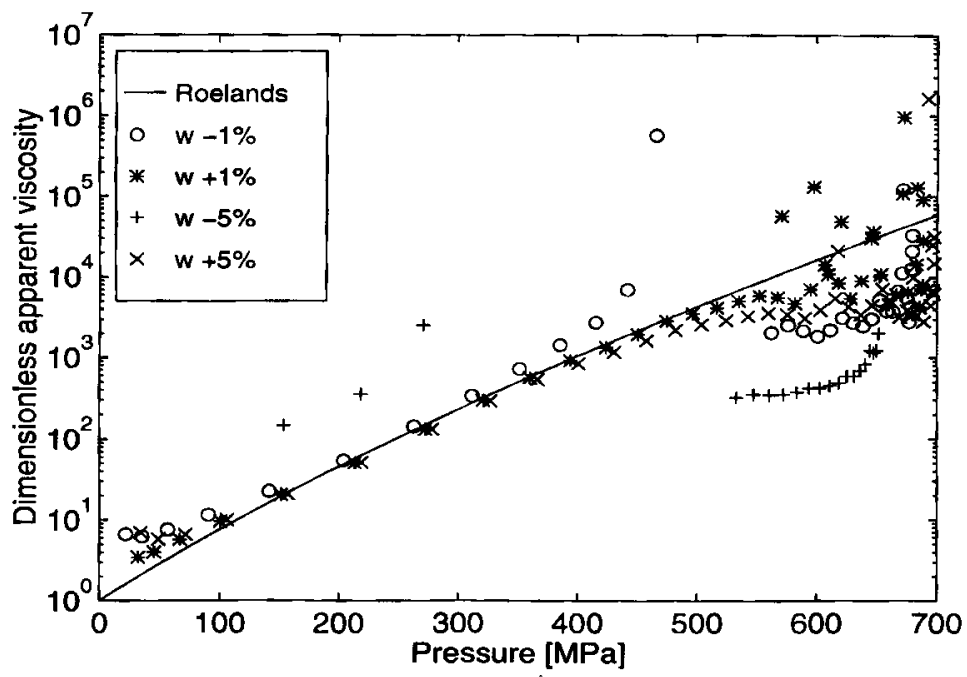

Fig. 7 Effect of error in load, $w$, on the dimensionless apparent viscosity

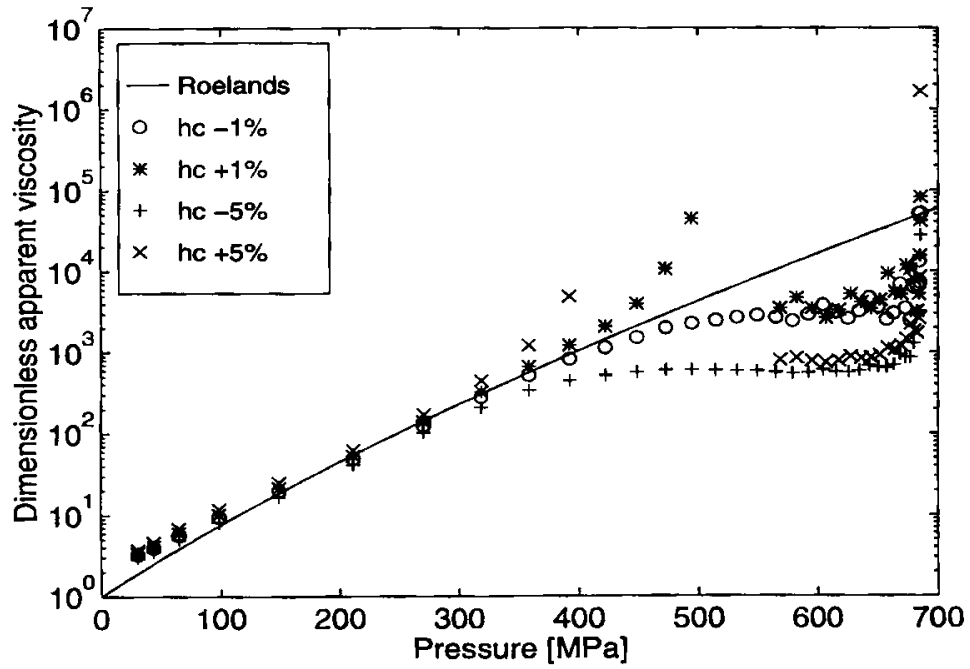

Fig. 8 Effect of error in the central film thickness on the dimensionless apparent viscosity

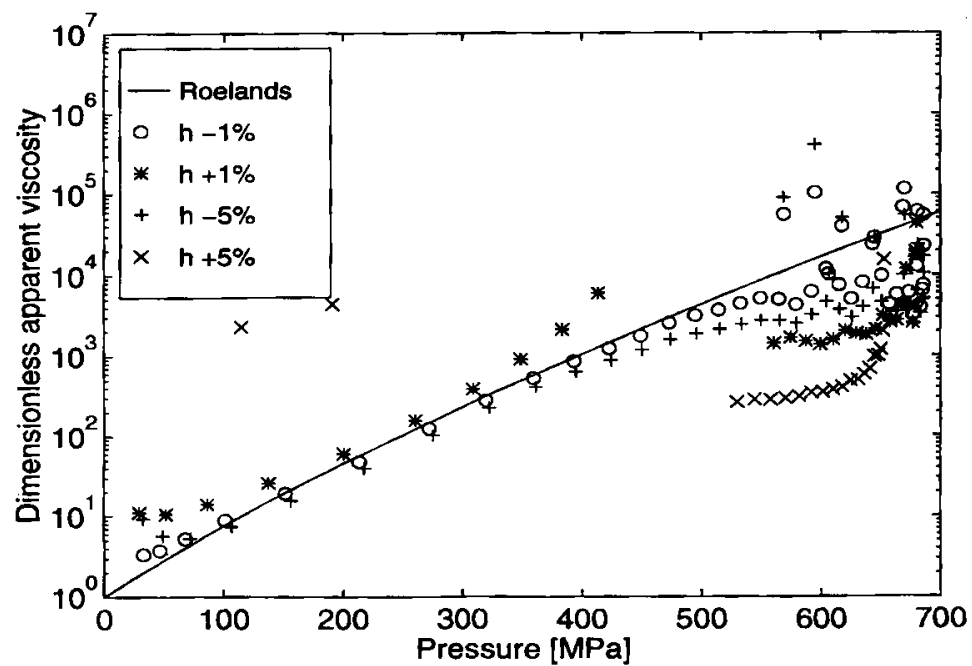

Fig. 9 Effect of error in the film thickness, $h$, on the dimensionless apparent viscosity 
change difference and refractive index, that is an error in the central film thickness and an overall error in the film thickness. As seen in Fig. 5c, the pressure distribution will deviate more from the exact pressure distribution if the film thickness is too large. This explains why larger errors in the dimensionless apparent viscosity occur in these cases.

Figure 10 shows the effect of a centring error. It is seen that the solution of the dimensionless apparent viscosity is more sensitive to a centring error in the $y$ direction than in the $x$ direction. It can be seen that the centring error should be less than 1 per cent of the Hertzian radius to get reliable results.

The resolution in the film thickness measurement seems to play an important role, as seen in Fig. 11. The pressure will be close to the exact pressure. However, due to the limited resolution, the Couette term of equation (5), $\partial(\rho h) / \partial x$, will contain large errors. The Couette term is very sensitive to this kind of error, and it can be seen that the resolution should be better than $1 \mathrm{~nm}$. Otherwise some smoothing algorithm should be used to get rid of the steps in the film thickness map.

Figures 12 and 13 show the effects from errors in the velocity sum and in the viscosity at ambient pressure. It is seen that these errors do not give as severe effects on the apparent viscosity as other errors. Figure 12 shows that errors in the velocity sum give rise to an error in the dimensionless apparent viscosity, which is more or less inversely proportional to the velocity sum error. This means that if $u$ is measured 1 per cent too large the dimensionless apparent viscocity will be approximately 1 per cent too small. Figure 13 shows that the viscosity at ambient pressure influences the apparent viscosity very little.

The effects of different errors on the apparent viscosity are large at high pressures. These errors do not, of course, always occur in isolation and combined errors will give even larger effects on the apparent viscosity.

Another source of error that has not been discussed is the thermal effect. The analysis presented here is isothermal, but the film thickness would decrease by a few per cent if inlet shear heating had been taken into account. Gupta et al. (13) gave an empirical formula for calculating the film thickness reduction due to inlet heating. This reduction is approximately 3 per cent for this case. It can thus be concluded that thermal effects may influence the apparent viscosity much, since an error in film thickness by $1-5$ per cent seems to give large errors in apparent viscosity (see Figs 8 and 9).

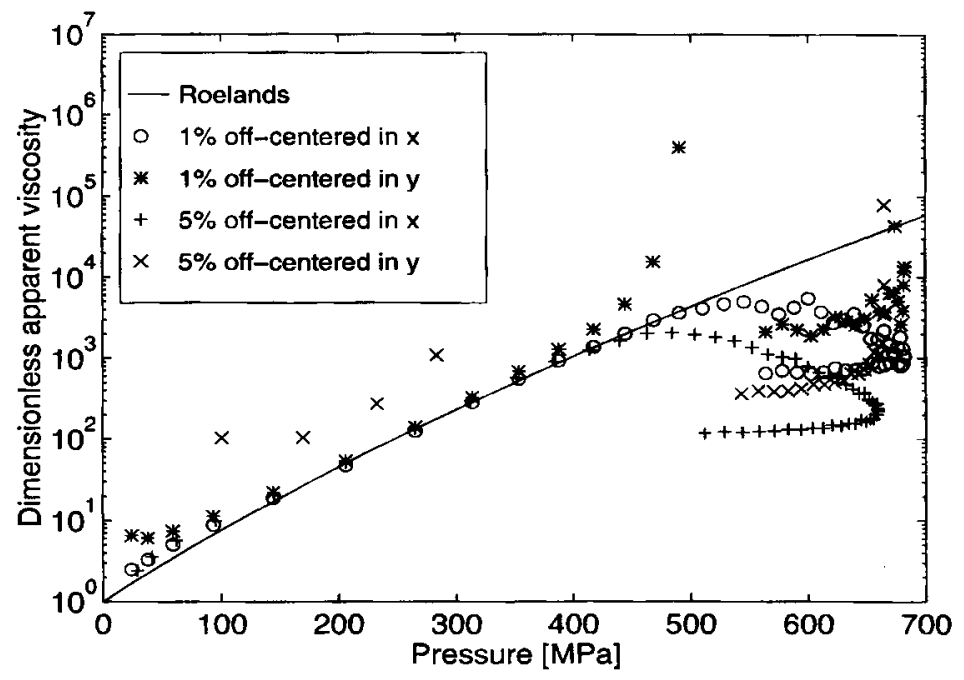

Fig. 10 Effect of error in centring on the dimensionless apparent viscosity

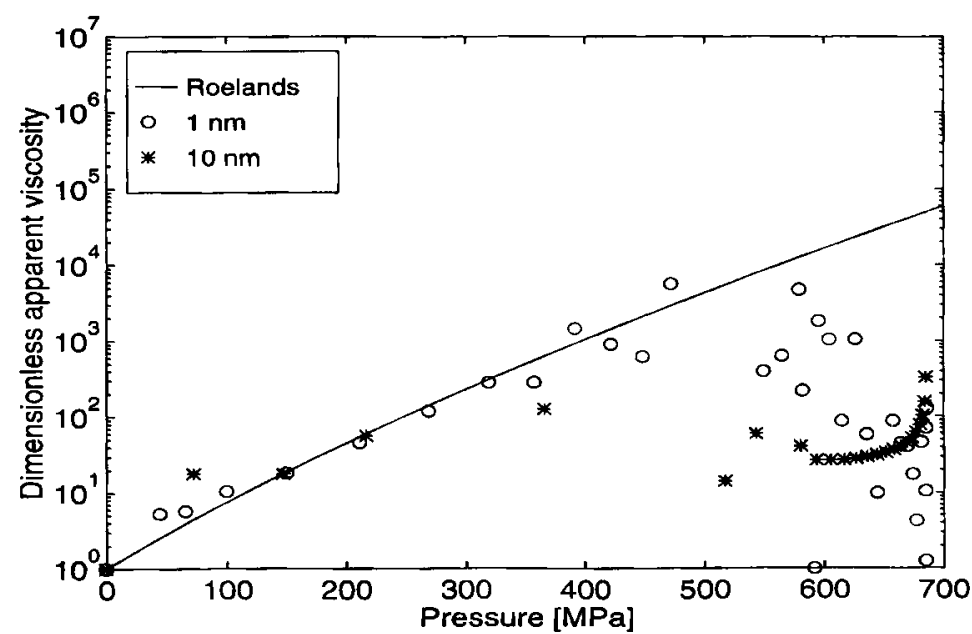

Fig. 11 Effect of truncation error in film thickness on the dimensionless apparent viscosity 


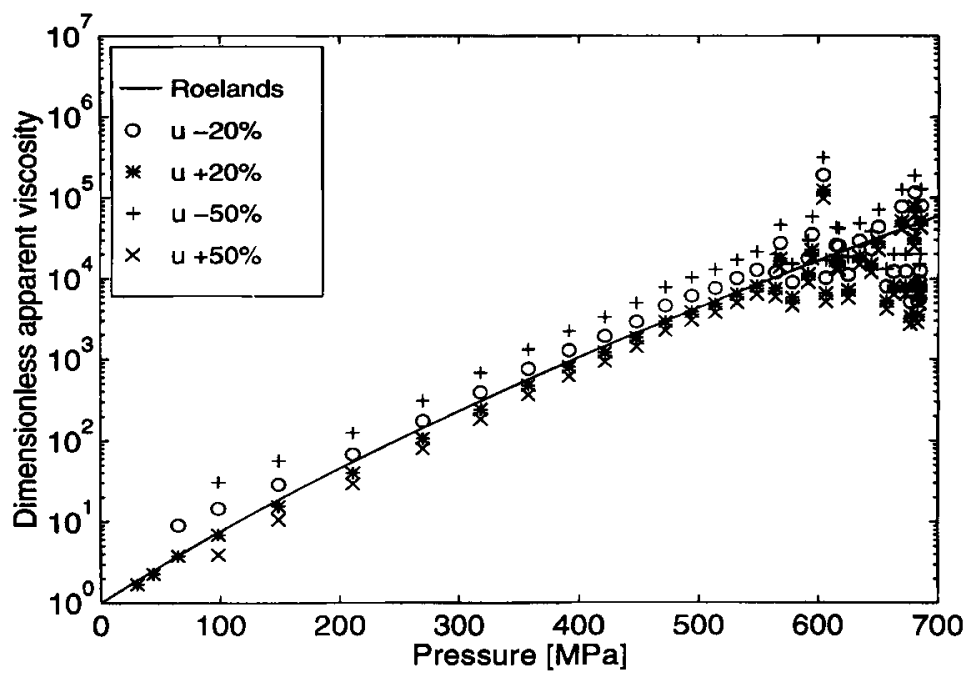

Fig. 12 Effect of error in the velocity sum, $u$, on the dimensionless apparent viscosity

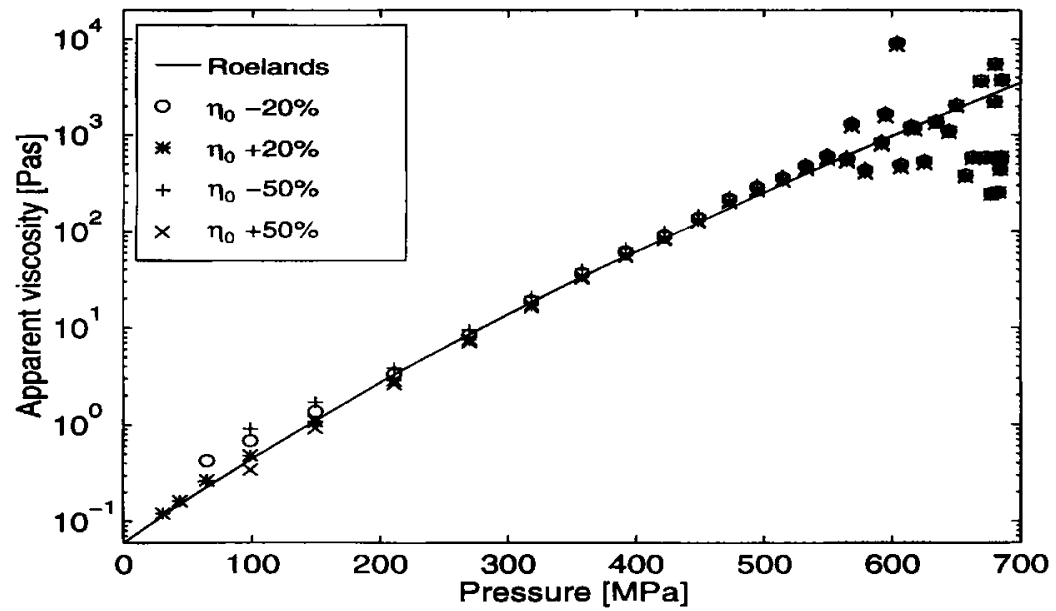

Fig. 13 Effect of error in the viscosity at ambient pressure, $\eta_{0}$, on the apparent viscosity

\section{CONCLUSIONS}

The conclusions from the present investigation are:

1. The pressure distribution in an EHD point contact can be solved by the use of inverse elasticity theory if the film thickness is known, from, for example, optical interferometry measurements.

2. The sensitivity on the solution of the pressure distribution to measurement errors has been investigated in this paper. The simulated errors which have been used (see Table 2) do not influence the pressure within the Hertzian region by more than a few per cent.

3. The apparent viscosity in an EHD point contact can be obtained from the Reynolds equation if the film thickness and the pressure are known.

4. Numerical problems make it difficult to compute apparent viscosity at the contact centre. Injection of film thickness data from fine grids to coarse grids makes it difficult to compute apparent viscosity at low pressure. In this case the apparent viscosity can thus only be found at pressures within approximately $100-500 \mathrm{MPa}$.
The following measurement accuracy is needed to obtain reliable results for the apparent viscosity:

5. The error in effective elastic modulus has to be within -5 and +1 per cent from the true effective elastic modulus.

6. The error in load has to be within -1 and +5 per cent from the true load.

7. The error in the lubricant's refractive index must give an overall error in film thickness which is less than \pm 1 per cent from the true film thickness. This error in film thickness corresponds to $\mathrm{a} \pm 1$ per cent error in refractive index.

8. The error in phase change due to reflection in the surfaces must give an error in central film thickness which is less than \pm 1 per cent of the true central film thickness, which in this case corresponds to an approximate error of \pm 10 per cent in $\Delta \phi$.

9. To compute the pressure, it is necessary to find the ball's curvature centre in the film thickness map. The centring error in the sliding direction has to be within 5 per cent of the Hertzian radius. The centring error in the perpendicular direction has to be within 1 per cent of the Hertzian radius. 
10. It is important to measure the film thickness with high resolution. The smallest detectable difference in film thickness must not exceed $1 \mathrm{~nm}$.

11. An error in the velocity sum gives an error in the dimensionless apparent viscosity which is inversely proportional to the velocity sum error.

12. The viscosity at ambient pressure has a small effect on the apparent viscosity.

13. Since combined errors are likely to occur, there may be a need of an even better accuracy than stated in conclusions 5 to 11 .

14. The film thickness reduction due to inlet heating should be at least less than 1 per cent.

\section{ACKNOWLEDGEMENTS}

The authors wish to acknowledge Professor Erik Höglund, Luleå University of Technology, for his assistance. They also wish to acknowledge the financial support of the COLDTECH Foundation.

\section{REFERENCES}

1 Cameron, A. and Gohar, R. Theoretical and experimental studies of the oil film in lubricated point contact. Proc. R. Soc., Ser. A, 1966, 291, 520-536.

2 Foord, C. A., Hammann, W. C. and Cameron, A. Evaluation of lubricants using optical elastohydrodynamics. ASLE Trans., 1968, 11, 31-43.
3 Paul, G. R. and Cameron, A. A high-pressure microviscometer based on refractive index. Proc. $R$. Soc., Ser. A, 1972, 331, 171-184.

4 Wong, P. L., Lingard, S. and Cameron, A. The high pressure impact microviscometer. STLE Tribology Trans., 1992, 35(3), 500 508.

5 Wong, P. L., Lingard, S. and Cameron, A. A simplified impact microviscometer. Tribology Int., 1992, 25(6), 363-366.

6 Wong, P. L., Lingard, S. and Cameron, A. High pressure velocity and shear response of oil using the rotating optical microviscometer. Presented at the Leeds-Lyon Symposium, 1994.

7 Gustafsson, L., Höglund, E. and Marklund, O. Measuring lubricant film thickness with image analysis. Proc. Instn Mech. Engrs, Part J, 1994, 208(J3), 199-205.

8 Åström, H. and Venner, C. H. Soap-thickener induced local pressure fluctuations in a grease-lubricated elastohydrodynamic point contact. Proc. Instn Mech. Engrs, Part J, 1994, 208(J3), 191-198.

9 Dowson, D. and Higginson, G. Elastohydrodynamic lubrication; the fundamentals of roller and gear lubrication, 1966 (Pergamon Press, Oxford).

10 Venner, C. H. and ten Napel, W. E. Multilevel solution of the elastohydrodynamically lubricated circular contact problem. Part 1: theory and numerical algorithm. Wear, 1992, 152, 351-367.

11 Venner, C. H. and ten Napel, W. E. Multilevel solution of the elastohydrodynamically lubricated circular contact problem. Part 2: smooth surface results. Wear, 1992, 152, 369-381.

12 Roelands, C. J. A. Correlation aspects of the viscositytemperature-pressure relationship of lubricating oils. $\mathrm{PhD}$ thesis, Technische Hogeschool Delft, The Netherlands (VRB, Groningen, The Netherlands), 1966.

13 Gupta, P. K., Cheng, H. S. and Forster, N. H. Visco-elastic effects in MIL-L-7808-type lubricant. Part I: analytical formulation. STLE Tribology Trans., 1992, 35(2), 269-274. 